AD-A062 314 FLORIDA STATE UNIV TALLAHASSEE DEPT OF STATISTICS LARGE SAMPLE ESTIMATES AND UNIFORM CONFIDENCE BOUNDS FOR THE FA--ETC(U) UNCLASSIFIED AUG 78 J SETHURAMAN. N D SINGPURWALLA NOO014-77-C-0263

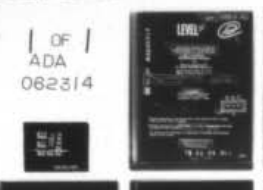
FSU-STATISTICS-M472

$$
\text { ARO }-13888 \cdot 14-M X
$$
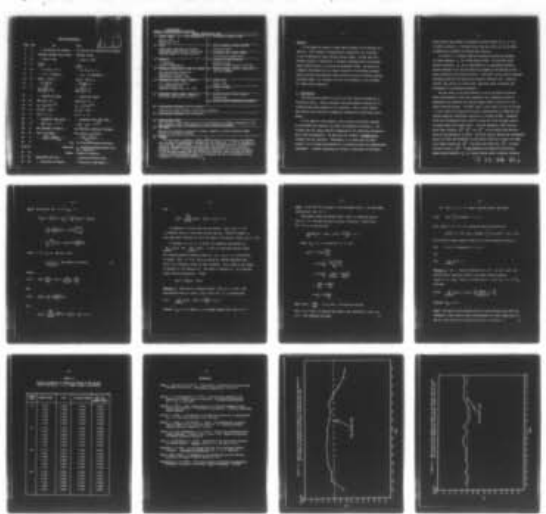

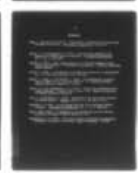

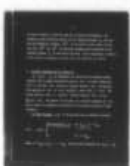
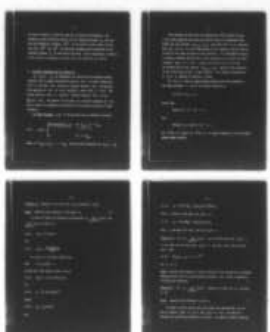
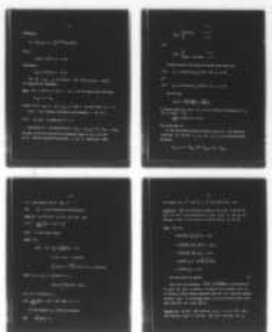

NL
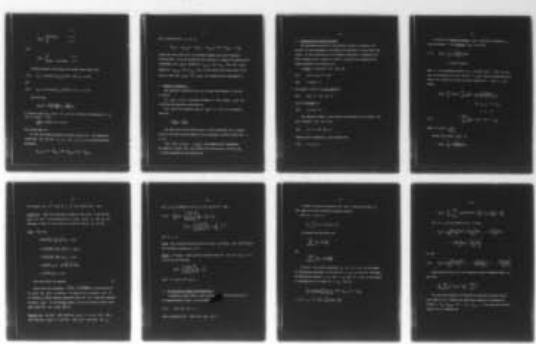

END

DATE
FILUED

379 


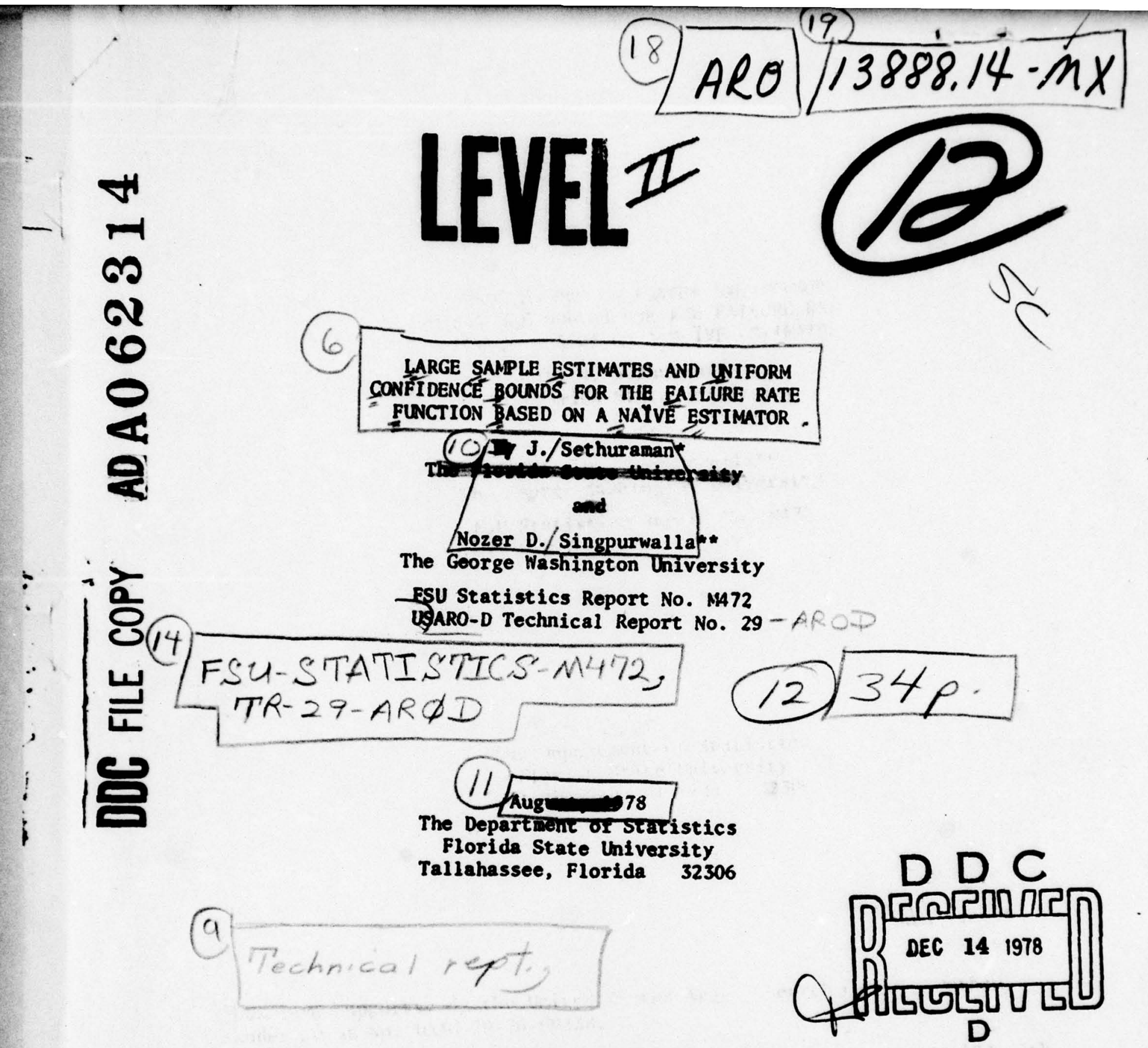
*Research supported by the United States Army Research Office, Durham,
under Grant No. DANG 29-76-60238.

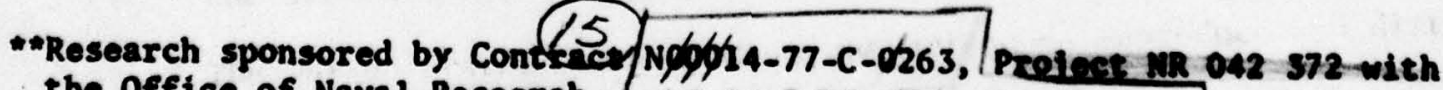
the Office of Naval Research. JDAAG29-76-6-\$口238

The United States Government is authorized to reproduce and distribute copies of this report.

$$
\begin{aligned}
& \text { DISTRIBUTYON STATENENT I } \\
& \text { Approved for public zelecse; } \\
& \text { Distribution Unlimited } \\
& \begin{array}{llll}
81204 & 081 .
\end{array} \\
& 400277
\end{aligned}
$$




\section{List of Corrections}

Page Line

For

Read

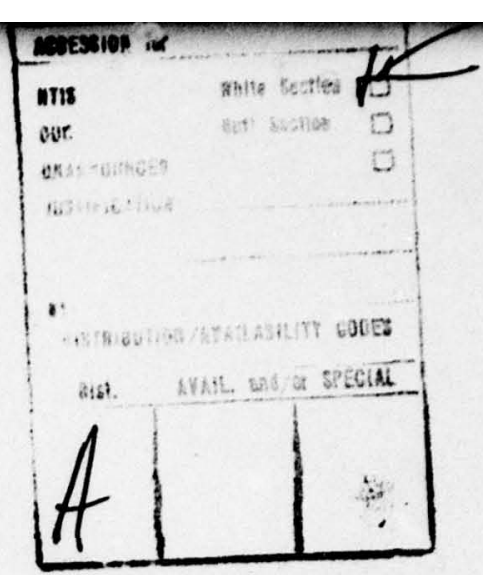

36

... the failure are studied ...

the failure rate functions are studied.

66

Standard extreme value theory..

Standard theory ...

$6 \quad 13$

given i that

... given $i$, that

614

$\frac{1}{n_{x}(x)} \cdots$

813

$\ldots 0 \leq w(u) \leq M<\infty$.

$\frac{1}{r_{n}(x)} \cdots$

95

... (i.e., satisfies...

$\ldots 0 \leq w(u) \leq c<\infty$.

$10 \quad 12$

$\mid R_{n}(x)-H_{n}(x) \leq \cdots$

... (i.e., it satisfies ...

122

$\ldots \int w \frac{x-s}{b_{n}} d[\ldots$

126

$$
\frac{1^{n}}{2 n b_{n} \bar{F}_{n}(K+A)}
$$

$\left|R_{n}(x)-H_{n}(x)\right| \leq \cdots$

... $\int w\left(\frac{x-3}{b_{n}}\right) d[\ldots$

$\frac{2 c}{2 n b_{b} \bar{F}_{n}(K+A)}$

$161 \quad \ldots$ replaced by $\bar{E}_{n}(s)$.

... replaced by $\bar{\xi}_{n}(x)$.

$1614 \operatorname{mx} E_{n}(x)$, we ...

$\left.1616 \quad B_{n}=c_{n} /(\lambda / w)\right)^{1 / 2}$,

$\max \left|\xi_{n}(x)\right|$, we ...

$B_{n}=c_{n} /(\lambda(w))^{1 / 2}$,

176 or $(W 1)-w(4) \ldots$

or (W1) - (W4) ...

179 for $0<x<\infty$.

for $0<2<\infty$.

$192 \quad \ldots$ assumption (B3) holds...

... assumption that (B3) holds...

$\left.20 \quad 3 \quad \ldots\left(\frac{2}{n}+a_{n}\right)\right\} \rightarrow e^{-2^{-2}}$,

206 are uniformly estimates...

2015 This $w$ satisfies...

226

279

$\ldots+\frac{x_{(1)}-x_{(1-1)}}{(n-1+1) U_{(1)}}+\ldots$

2713,14

$27 \quad 16$

$\left.\ldots\left(\frac{2}{\beta_{n}}+\alpha_{n}\right)\right\} \rightarrow e^{-2 e^{-2}}$,

are uniformly consistent estinates

This window w satisfies...

$\ldots+\frac{x_{(i)}-x_{(i-1)}}{U(i)}+\ldots$

Add: The George Washington University.

Underline: 2 . Wahrscheinlichkeitstheorie und Verw. Goblete 32

$27 \quad 21$

generallzed end rate...

Rewove: Program in Logistics, 
REPORT DOCUMENTATION PAGE

\begin{tabular}{l|l|l|l}
\hline 1. REPORT NUMIBER & 2. GOVT ACCESSION NO. & 3. RECIPIENT'S CATALOG NUKBER
\end{tabular}

FSU No. 1442

USARO-D No. $29 \checkmark$

4. TITLE

LARGE SAMPLE ESTIMATES AND UNIFORII CONFIDENCE BOUNDS FOR THE FAILURE RATE

FUNCTION BASED ON A NAÏVE ESTINIATOR

7. AUTHOR(s)

J. Sethuraman

Nozer D. Singpurwalla

9. PERFORNING ORGANIZATION NAVE AND ADDRESS

The Florida State University

Department of Statistics

Tallahassee, Florida 32306

11. CONTROLLING OFFICE NAME \& ADDRESS

U. S. Army Research Office

Post Office Box 12211

Research Triangle Park, NC 27709

14. MONITORING AGENCY NAME \& ADDRESS (if different from Controlling office)
5. TYPE OF REPORT \& PERIOD COVERED

Technical Report

6. PERFORMING ORG. REPORT NUMBER

FSU Statistics Report $M 472$

8. CONTRACT OR GRANT NUMBER(s)

DAAG29 -76-G0238

N00014-77-C-0263 Project NR 042372

10. PROGRAN ELER-ENT, PROJECT, TASK AREA \&

WORK UNIT NUMBERS
12. REPORT DATE

August, 1978

13. NUMBER OF PAGES

27

15. SFCURITY CLASS (of this report)

UNCLASSIFIED

15a. DECLASSIFICATION/DOWNGRADING SCHEDULE

16. DISTRIBUTION STATELENT (of this report)

Approved for public release; distribution unlimited.

17. DISTRIBUTION STATERENT (of the abstract)

18. SUPPLEVIENTARY NOTES

The findings in this report are not to be construed as an official Department of the Army position, unless so designated by other authorized documents.

19. KEY WORDS

failure rate function, consistent estimate, asymptotic uniform confidence bands, axima of stationary processes

20. ABSWRACT

In this paper we propose a simple naive estimator of the failure rate function. This estimate is asymptotically unbiased but not consistent. It can be smoothed by using any band limited window. We show that this smoothed estimate is equivalent to estinates obtainable from the modified sample hazard function, as in Rice and Rosenblatt (1976). We obtain the asymptotic distribution of the global deviation of the smothed estimate from the failure rate function, which can then be used to construct uniform confidence bands. We illustrate the rate of convergence of our estimator by a Monte-Carlo simulation. 


\section{Abstract.}

In this paper we propose a simple naive estimator of the failure rate function. This estimate is asymptotically unbiased but not consistent. It can be smoothed by using any band limited window. We show that this smoothed estimate is equivalent to estimates obtainable from the modified sample hazard function, as in Rice and Rosenblatt (1976). We obtain the asymptotic distribution of the global deviation of the smoothed estimate from the failure rate function, which can then be used to construct uniform confidence bands. We illustrate the rate of convergence of our estimator by a Monte-Carlo simulation.

\section{Introduction.}

The failure rate function is one of the most important parameters in reliability theory. Several parametric and non-parametric methods for its estimation have been proposed in the literature. Nost of these methods make specific and restrictive assurptions concerning the underlying distribution.

In this paper we first propose a very intuitive (and naïv) estimate of the failure rate function, and study its properties in Section 2 . Our estimate does not require specific assumptions on the underlying distribution and is thus non-paranetric. We show that our estimate is asyeptotically unbiased, but not consistent. Purtherwore, it is shown that the naive estimate of the failure rate function at two distinct points are asymptotically independent. A paradox concerning the estimate, reminiscent of the famous 
inter-arrival time paradox illustrated by Feller [(1966) Vol. 2, p. 11], is shown in Section 3. The above facts limit any direct use of the naïve estimator for estimating the failure rate function.

In Section 4, we propose smoothed estimators obtained by averaging the naive estimator $r_{n}$ by a band-limited window. We show that these smoothed estimators $\overline{\mathbf{r}}_{\mathrm{n}}$ can be approximated by an appropriate Gaussian process (Theorem 4.5), and thus obtain the asymptotic distribution of the global deviation on any finite interval. This result can be used to construct confidence bands for the failure rate function (Theorem 4.10). Section 5 contains some results of a Monte-Carlo experiment which illustrates the performance of our smoothed estimators.

The main steps in the proof Theorem 4.5 may be described as follows. Bickel and Rosenblatt (1973) have pioneered a new technique of proof to approximate the normalized and centered sample density function by a stationary Gaussian process. The basic step in their proof is a use of the then ailable result of Breiman and brillinger approximating the normalized and centered empirical distribution function by a Brownian bridge. Rosenblatt (1976) has strengthened these results by using the recent stronger results of Koml6s, Major and Tusnady (1975). Rice and Rosenblatt (1976) have proposed three estimates $h_{n}^{(1)}, h_{n}^{(2)}$, and $h_{n}^{(3)}$ of the failure rate function which are non-parametric in nature. They have directly applied the strengthened results of Bickel and Rosenblatt on the density function to obtain the asymptotic global results for $h_{n}^{(1)}$. They have also shown that $h_{n}^{(2)}$ is asymp-. totically close to $h_{n}^{(3)}$. By approximating the normalized and centered sample hazard functions $h_{h}$ by a Weiner process under a monotone transform

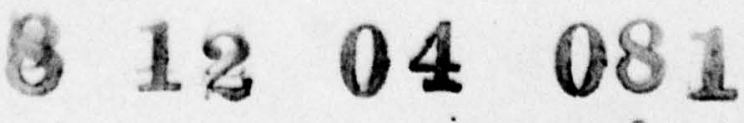


of time, we obtain, in much the same way as Bickel and Rosenblatt, the asymptotic global deviation results for our smoothed estimate $\overline{\mathbf{r}}_{\mathbf{n}}$ and the Rice and Rosenblatt estimator $h_{n}^{(3)}$. In the course of this proof, we also show that $h_{n}^{(2)}$ and $h_{n}^{(3)}$ are uniformly asymptotically equivalent to our smoothed estimate $\bar{r}_{n}$ on each finite interval. Other nonparametric estimates of the failure are studied in Shaked (1978) and Ahnad and Lin (1977).

2. The Naïve Estimate and its Properties.

Let $x_{1}, x_{2}, \ldots, x_{n}$ be independent and identically distributed random variables with a common distribution function $F(x)$. We shall assume that $F(0)=0$ and that $F(x)$ possesses a density function $f(x)$. The failure rate function of $F(x)$ is $r(x)=f(x) / \bar{F}(x)$ where $\bar{F}(x)=1-F(x)$. The hazard function $H(x)$ is $-\log \bar{F}(x)$ (defined whenever $\bar{F}(x)>0$ ) and : $\frac{d}{d x} H(x)=r(x)$. The purpose of this paper is to provide estimates of $r(x)$ and to obtain the asymptotic distribution of the global deviation of these estimates.

The naive estimate $r_{n}(x)$ of the failure rate is defined as follows:

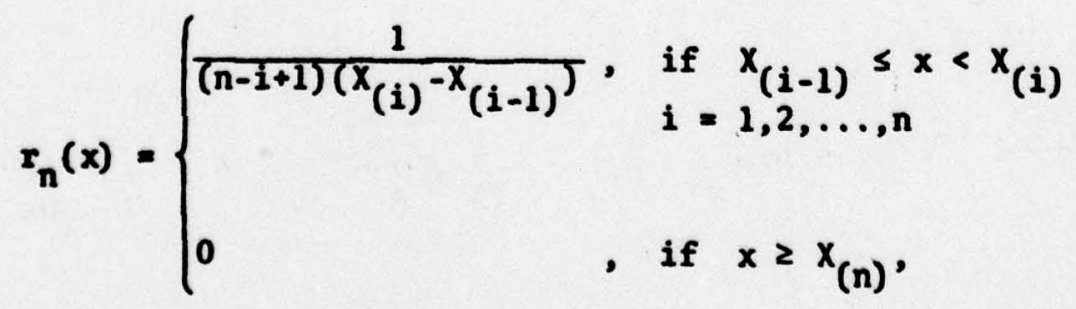

where $0=x_{(0)} \leq x_{(1)} \leq \ldots \leq x_{(n)}$ are the order statistics of $x_{1}, x_{2}, \ldots, x_{n}$. 
This estimate has been used by Singpurwalla (1975) wherein he used a time series approach for analyzing failure rates in contaminated data. Before the time interval $\left[X_{(i-1)}, X_{(i)}\right)$ there have been $(i-1)$ failures; thus only $(n-i+1)$ are still functioning in this interval, and one item is to fail at the right end point of this interval. It is therefore intuitive to assign a constant failure rate in this interval and to define the naive estimator $\mathbf{r}_{n}(x)$ as $i_{i}(2.1)$. Another notivation : or (2.1) is that the failure rate in the interval $\left[x_{(i-1)}, x_{(i)}\right)$ should be the reciprocal of the 'total time on test' in that interval. The limiting distribution of $r_{n}(x)$ is obtained in Theorem 2.1 below.

Let $G(\alpha ; \lambda)$ denote a gamma random variable with scale parameter $\alpha$ and shape parameter $\lambda$; that is the density function is

$$
a^{\lambda} e^{-x \alpha} x^{\lambda-1} / \Gamma(\lambda), x>0
$$

Notice that

$$
E(G(\alpha ; \lambda))=\lambda / \alpha \text { for } \lambda>0
$$

and

$$
E(1 / G(\alpha ; \lambda))=\alpha /(\lambda-1) \text { for } \lambda>1
$$

Let $G^{-1}(\alpha ; \lambda)=1 / G(\alpha ; \lambda) ; G^{-1}(\alpha ; \lambda)$ is usually referred to as the inverse ganm randon variable. 
Theorem 2.1.

(i) $P\left(1 / r_{n}(x) \leq a\right)+\int_{0}^{a} e^{-y r(x)} y(r(x))^{2} d y$;

that is

$$
1 / r_{n}(x) \rightarrow G(r(x), 2) \text { in law. }
$$

Consequent ly

$$
r_{n}(x) \rightarrow G^{-1}(r(x), 2) \text { in law. }
$$

(ii) Let $x_{1}, x_{2}, \ldots, x_{k}$ be distinct. Then $\left\{r_{n}\left(x_{1}\right), r_{n}\left(x_{2}\right), \ldots, x_{n}\left(x_{k}\right)\right\}$ are asymptotically independent.

Proof. Fix $x$, and let $0<F(x)<1$. Let $i$ be the random suffix satisfying

$$
x_{(i-1)} \leq x<x_{(i)}
$$

wherein, we set $x_{(0)}=0$, and $x_{(n+1)}=\infty$; thus $i$ can take values $1,2, \ldots, n$.

Since $i$ has a binomial distribution with parameters $n$ and $F(x)$,

$$
\frac{i}{n} \rightarrow F(x) \text {, in probability as } n \rightarrow \infty \text {. }
$$

Conditional on $i$, the distribution of $\left(x_{(1)}, \ldots, x_{(i-1)}\right)$ and $\left(x_{(i)}, \ldots, x_{(n)}\right)$ are those of the order statistics of independent sample os size (1-1) and $(n-i+1)$ frow the truncated distributions $F_{x}$ and $F^{x}$, respectively, where 


$$
F_{x}(y)= \begin{cases}F(y) / F(x) & y \leq x \\ 1 & y>x\end{cases}
$$

and

$$
F^{x}(y)= \begin{cases}0 & y \leq x \\ (F(y)-F(x)) / \bar{F}(x) & y>x .\end{cases}
$$

Standard extreme value theory for extreme values shows that

$$
A_{n}=(i-1) f(x)(x-x(i-1)) / F(x) \rightarrow G(1,1) \text { in law }
$$

and

$$
B_{n}=(n-i+1) f(x)\left(X_{(i)}-x\right) / \bar{F}(x) \rightarrow G(1,1) \text { in law. }
$$

Note now that

$$
1 / r_{n}(x)=\frac{n-i+1}{i-1} \frac{F(x)}{f(x)} A_{n}+\frac{\bar{F}(x)}{f(x)} B_{n} .
$$

It follows from (2.2), (2.3), (2.4) and the conditional independence of $A_{n}$ and $B_{n}$ given $i$ that

$$
\frac{1}{n_{r}(x)}+G(r(x), 2) \text {, in law. }
$$

This proves part (i).

We will now briefly indicate the proof of part (ii). For simplicity, sssume that $k=2$, and that $x_{1}<x_{2}$. Let $i_{1} \leq i_{2}$ be the random suffixes satisfying

$$
x_{\left(i_{1}-1\right)} \leq x_{1}<x_{\left(i_{1}\right)} \text { and } x_{\left(i_{2}-1\right)} \leq x_{2}<x_{\left(i_{2}\right)} \text {. }
$$


Then, conditional on $i_{1}$ and $i_{2}$,

$$
\left.{ }_{(1)}, \ldots, x_{\left(i_{1}-1\right)}\right), \quad\left(x_{\left(i_{1}\right)}, \ldots, x_{\left(i_{2}-1\right)}\right) \text {, and }\left(x_{\left(i_{2}\right)}, \ldots, x_{(n)}\right)
$$

become the order statistics in independent samples from three truncated distributions. Si:ce the minimum and the maximum of a sample are asymptotically independent, and $r_{n}\left(x_{1}\right)$ depends on $x_{\left(i_{1}-1\right)}$ and $x_{\left(i_{1}\right)}$ only, and $r_{n}\left(x_{2}\right)$ depends on $x_{\left(i_{2}-1\right)}$ and $x_{\left(i_{2}\right)}$ only, we can imitate the earlier part of our proof to show that $r_{n}\left(x_{1}\right)$ and $r_{n}\left(x_{2}\right)$ are asymptotically independent. $\mid 1$.

\section{Remarks on Theorem 2.1.}

The important conclusions that can be drawn from Theorem 2.1 are the following:

(i) $r_{n}(x)$ is not a consistent estimate of $r(x)$; indeed, $r_{n}(x)$ has a limiting non-degenerate distribution.

(ii) While the asymptotic mean of $r_{n}(x)$ is $r(x)$, we can actually show that

$$
E \frac{1}{r_{n}(x)} \rightarrow 2 \frac{1}{r(x)}
$$

The factor of 2 in the above result is quite surprising, and is reminiscent of the inter-arrival paradox so well explained in Feller [(1966) Vol. 2, p. 11].

(iii) Since $\left(r_{n}\left(x_{1}\right), \ldots, r_{n}\left(x_{k}\right)\right)$ are asymptotically independent, the graph of $\left\{r_{n}(x), x \geq 0\right\}$ will exhibit wild fluctuations, and will not ha good estimator of the failure rate. 
4. A Smoothing of the Naïve Estimate.

The phenomenon described in the previous section is similar to the behavior of the periodogram in estimating the spectrum of a stationary time series. We shall therefore use the standard technique of 'smoothing' the naïve estimate with a 'window' to obtain a consistent and asymptotically normal estimate of the failure rate.

A window is a function $w(u)$ such that

$$
w(u)=w(-u) \geq 0 \text {, and }
$$

$$
\int w(u) d u=1 .
$$

The window is said to be band-1imited if

$$
w(u)=0, \text { for }|u| \geq A \text {, }
$$

and to be bounded, if

$$
0 \leq w(u) \leq M<
$$

The smoothed estimatcy of the failure rate depends on the window $w(u)$ and a sequence $\left\{b_{n}\right\}$ sati fying

$$
b_{n}+0 \text {, and } n b_{n}+\infty \text {. }
$$

Without loss of generality, we may assume that

$$
0<b_{n} \leq A \text {. }
$$


He define the smoothed estimator $\bar{r}_{n}(x)$ obtained by smoothing $r_{n}$ with the window $w$ and bandwidth $2 b_{n} A$ as follows:

(4.1) $\quad \bar{r}_{n}(x)=\frac{1}{b_{n}} \int w\left(\frac{x-s}{b_{n}}\right) r_{n}(s) d s$

$=\int w(s) r_{n}\left(x-b_{n} s\right) d s$.

When $w$ is a bandlimited window (i.e., satisfies (iv3)), $\bar{r}_{n}(x)$ will be used as an estimate of $r(x)$ only for $x \geq b_{n} A$, since the smoothing being done in (4.1) does not include the whole bandwith of $w$ when $x<b_{n} A$. Define

$$
\begin{array}{r}
R_{n}(x)=\int_{0}^{x} r_{n}(y) d y=\sum_{j=1}^{i-1} \frac{1}{n-j+1}+\frac{x-x_{(i-1)}}{(n-i+1)\left(x_{(i)} x_{(i-1)}\right)} \\
\text { for } x_{(i-1)} \leq x<x_{(i)}, \\
i=1, \ldots, n,
\end{array}
$$

$$
=\sum_{j=1}^{n F_{n}(x)} \frac{1}{n-j+1}+\theta_{n}(x) \text { for } x<x_{(n)}
$$

where $0 \leq \theta_{n}(x) \leq \frac{1}{n \bar{F}_{n}(x)}$.

We may now re-write $\overline{\mathbf{r}}_{n}(x)$ as

$$
\bar{r}_{n}(x)=\frac{1}{b_{n}} \int w\left(\frac{x-s}{b_{n}}\right) d R_{n}(x) .
$$


The following lemma shows that $R_{n}(x)$ and $H_{n}(x)=-\log \bar{F}_{n}(x)$ (defined only for $x<x_{(n)}$ ) are uniformly close to each other in bounded intervals.

Lemma 4.1. Fix $K<\infty$; then

$$
\sup _{0 \leq x \leq K}\left|R_{n}(x)-H_{n}(x)\right| \leq \frac{3}{2 n \bar{F}_{n}(K)}
$$

if $\bar{F}_{n}(K)>0$; i.e., if $K<X_{(n)}$.

Proof. We shall use the elementary inequality

$$
|x+\log (1-x)| \leq \frac{x^{2}}{2(1-x)} \text { for } 0 \leq x<1
$$

Note that

(4.4) $\quad H_{n}(x)=-\log \bar{F}_{n}(x)=\sum_{j=1}^{n F_{n}(x)} \log \frac{n-j+1}{n-j}=-\sum_{j=1}^{n F_{n}(x)} \log \left(1-\frac{1}{n-j+1}\right)$.

From equations (4.2) and (4.4), if $x \leq k<X_{(n)}$,

$$
\begin{aligned}
\mid R_{n}(x)-H_{n}(x) & \leq \sum_{j=1}^{n F_{n}(x)}\left|\frac{1}{n-j+1}+\log \left(1-\frac{1}{n-j+1}\right)\right|+\frac{1}{n \bar{F}_{n}(x)} \\
& ={ }_{n-n F_{n}(x)+1}^{n}\left|\frac{1}{k}+\log \left(1-\frac{1}{k}\right)\right|+\frac{1}{n \bar{F}_{n}(x)}
\end{aligned}
$$




$$
\begin{aligned}
& \leq \sum_{n-n F_{n}^{n}(K)+1}^{n} \frac{1}{2}\left(\frac{1}{k}\right)^{2} \frac{1}{1-\frac{1}{n-n F_{n}(K)+1}}+\frac{1}{n \bar{F}_{n}(K)} \\
& \leq \frac{1}{2} \frac{1}{n-n F_{n}(K)+1} \frac{n-n F_{n}(K)+1}{n-n F_{n}(K)}+\frac{1}{n \bar{F}_{n}(K)} \\
& =\frac{3}{2} \frac{1}{n \bar{F}_{n}(K)} .
\end{aligned}
$$

Rice and Rosenblatt (1976) have introduced a class of estimators $h_{n}^{(3)}(x)$, where

$$
h_{n}^{(3)}(x)=\frac{1}{b_{n}} \int w\left(\frac{x-s}{b_{n}}\right) d H_{n}(s) .
$$

In Lemma 4.2 we will show that our smoothed estimator $\bar{r}_{n}(x)$ is uniformly close to $h_{n}^{(3)}(x)$ on bounded intervals.

Lemma 4.2. Let $w$ be a bounded band limited window; that is, w satisfies (w1), (w2), (w3) and (w4). Let w also satisfy

$$
\int\left|w^{\prime}(x)\right| d x \leq c<\infty,
$$

where $w^{\prime}$ is the derivative of $w$.

Let the constants $\left(b_{n}\right\}$ satisfy $(B 1)$ and $(B 2)$, and let $k<\infty$. Then

$$
\sup _{0 \leq x \leq K}\left|\bar{x}_{n}(x)-h_{n}^{(3)}(x)\right| \leq \frac{3 c}{2 n b_{n} \bar{F}_{n}(K+A)}
$$

whenever $K+A \leq X(n)$. 


$$
\begin{aligned}
& \leq \sum_{n-n F_{n}(K)+1}^{n} \frac{1}{2}\left(\frac{1}{k}\right)^{2} \frac{1}{1-\frac{1}{n-n F_{n}(K)+1}}+\frac{1}{n \bar{F}_{n}(K)} \\
& \leq \frac{1}{2} \frac{1}{n-n F_{n}(K)+1} \frac{n-n F_{n}(K)+1}{n-n F_{n}(K)}+\frac{1}{n \bar{F}_{n}(K)} \\
& =\frac{3}{2} \frac{1}{n \bar{F}_{n}(K)} .
\end{aligned}
$$

Rice and Rosenblatt (1976) have introduced a class of estimators $h_{n}^{(3)}(x)$, where

$$
h_{n}^{(3)}(x)=\frac{1}{b_{n}} \int w\left(\frac{x-s}{b_{n}}\right) d H_{n}(s)
$$

In Lemma 4.2 we will show that our smoothed estimator $\bar{r}_{n}(x)$ is uniformly close to $h_{n}^{(3)}(x)$ on bounded intervals.

Lemma 4.2. Let $w$ be a bounded band limited window; that is, w satisfies $\left(w_{1}\right),\left(w_{2}\right),\left(w_{3}\right)$ and (w4). Let $w$ also satisfy

$$
\int\left|w^{\prime}(x)\right| d x \leq c<\infty \text {, }
$$

where $w^{\prime}$ is the derivative of $w$.

Let the constants $\left\{b_{n}\right\}$ satisfy $(B 1)$ and $(B 2)$, and let $K<\infty$. Then

$$
\text { (4.5) } \sup _{0 \leq x \leq K}\left|\bar{r}_{n}(x)-h_{n}^{(3)}(x)\right| \leq \frac{3 c}{2 n b_{n} \bar{F}_{n}(K+A)}
$$

whenever $K+A \leq X(n)$. 
Proof: Notice that, for $x \leq k \leq x_{(n)}-A$

$$
\begin{aligned}
\mid \bar{r}_{n}(x) & -h_{n}^{(3)}(x)|=| \frac{1}{b_{n}} \int w \frac{x-s}{b_{n}} d\left[R_{n}(x)-H_{n}(s)\right] \mid \\
\leq & \left|\frac{1}{b_{n}} w\left(\frac{x-s}{b_{n}}\right)\left[R_{n}(s)-H_{n}(s)\right]\right| \begin{array}{l}
x+b_{n} A \\
x-b_{n}^{A}
\end{array} \mid \\
& +\frac{1}{b_{n}^{2}} \int_{0}^{K+A}\left|R_{n}(s)-H_{n}(s)\right|\left|w^{\prime}\left(\frac{x-s}{b_{n}}\right)\right| d s,
\end{aligned}
$$

(since $x \leq k, b_{n} \leq A$, and $x+b_{n} \leq K+A$ )

$$
5 \frac{1}{2 n b_{n} \bar{F}_{n}(K+A)} \text { from Lemma } 4.1 \text { and (W5). }
$$

Define

(4.6)

$$
B(x)=\frac{F(x)}{\bar{F}(x)}, \quad B^{\prime}(x)=\frac{f(x)}{[\bar{F}(x)]^{2}}=\frac{r(x)}{\bar{F}(x)}
$$

and

(4.7)

$$
r_{n}^{*}(x)=\frac{1}{b_{n}} \int w\left(\frac{x-s}{b_{n}}\right) d H(s) .
$$

Let

$$
\xi_{n}(x)=\frac{\sqrt{n b_{n}}}{\sqrt{B^{\prime}(x)}}\left(h_{n}^{(3)}(x)-r_{n}^{*}(x)\right), \quad b_{n} A \leq x \leq k,
$$


and

$$
\bar{\xi}_{n}(x)=\frac{\sqrt{n b_{n}}}{\sqrt{B^{\prime}(x)}}\left(\bar{r}_{n}(x)-r_{n}^{*}(x)\right), \quad b_{n} A \leq x \leq k .
$$

In Theorem 4.4, we will show that the process $\left\{\xi_{n}(x), b_{n} A \leq x \leq k\right\}$ is uniformly close to a stationary Gaussian process. Because of Lemma 4.2, this same result (Theorem 4.5) will also apply to the process $\left\{\bar{\xi}_{n}(x), b_{n} A \leq x \leq K\right\}$.

In Theorems 4.6 , and 4.7 , we obtain the asymptotic distribution of

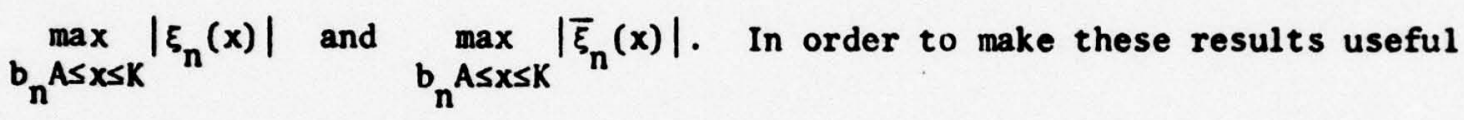
for obtaining untform confidence bands for $r(x), b_{n} A \leq x \leq k$, we will have to replace $r_{n}^{*}(x)$ by $r(x)$ and also replace any unknown quantities that enter in the asymptotic theory by their estimates. This is done in two stages, in Theorem 4.9 and Theorem 4.10. The result in Theorem 4.10 is in the most useful form for applications. Define

$$
Z_{n}(x)=\sqrt{n}\left(H_{n}(x)-H(x)\right) .
$$

Theorem 4.3. There exists a Gaussian process $\{Z(x), 0 \leq x \leq K+A\}$ with mean function zero and $E(Z(x), Z(y))=B(x)$ for $x \leq y$, and such that

$$
\sup _{0 \leq x \leq K+A}\left|z_{n}(x)-z(x)\right|=\frac{D \log n}{\sqrt{n}} \text { w.p. } 1
$$

whenever $X_{(n)}>K+A$, where $D$ is a random variable with $P(D<\infty)=1$. 
Proof: In the rest of this paper we use the generic name $D$ for any random variable with $P(D<\infty)=1$.

From Komlos, Major and Tusnady (1975), there is a Gaussian process $\{Y(x), 0 \leq x\}$ with mean function zero and $E(Y(x) Y(y))=F(x)(1-F(y))$ for $0 \leq x \leq y$, and such that

$$
\sup _{0 \leq x}\left|\sqrt{n}\left(F_{n}(x)-F(x)\right)-Y(x)\right|=\frac{D \log n}{\sqrt{n}} \text { w.p. } 1 \text {. }
$$

Since $X_{(n)}>K+A$, we have for $0 \leq x \leq K+A$,

$$
\begin{aligned}
Z_{n}(x) & =-\sqrt{n} \log \frac{\bar{F}_{n}(x)}{\bar{F}(x)} \\
& =-\sqrt{n} \log \left(1+\frac{\bar{F}_{n}(x)-\bar{F}(x)}{\bar{F}(x)}\right) \\
& =-\sqrt{n} \log \left(1-\frac{Y(x)}{\sqrt{n} \bar{F}(x)}+\frac{D \log n}{n \bar{F}(x)}\right) \\
& =\frac{Y(x)}{\bar{F}(x)}+\frac{D \log n}{\sqrt{n}} \\
& =Z(x)+D \frac{\log n}{\sqrt{n}}
\end{aligned}
$$

where $Z(x)=\frac{Y(x)}{\bar{F}(x)}, 0 \leq x \leq K+A$. It is easy to see that $\{Z(x), 0 \leq x \leq K+A\}$ is Gaussian with mean 0 , and $E(Z(x) Z(y))=B(x)$ for $x \leq y$. This completes the proof. 
Let $\{W(s),-\infty<s<\infty\}$ denote a Weiner process, and define

$$
\zeta(\theta)=\int_{-\infty}^{\infty} w(\theta-t) d w(t), \quad 0 \leq \theta<\infty .
$$

Then $\{\zeta(\theta), 0 \leq \theta<\infty\}$ is a stationary Gaussian process with

$$
E(\zeta(\theta))=0 \text { and } E(\zeta(\theta+\delta) \zeta(\theta))=\int_{-\infty}^{\infty} w(\delta+t) w(t) d t=\rho(\delta) \text { (say). }
$$

We now need to impose further conditions on the distribution function $F$ :

$$
B^{\prime \prime}(x) \text { is bounded on } 0 \leq x \leq K \text {, }
$$

and

$$
\inf _{0<x \leq K+A} B^{\prime}(x)>0
$$

Theorem 4.4. Let $w$ satisfy conditions (W1)-(W4). Let (B1), (B2), (F1) and (F2) hold. Then there exists a stationary Gaussian process $\left\{\zeta_{n}(\theta), A \leq \theta \leq K / b_{n}\right\}$ which is a restriction of $\{\zeta(\theta)\}$ for $A \leq \theta \leq K / b_{n}$, such that

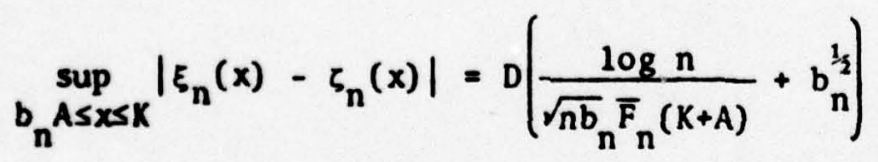

whenever $X_{(n)}>K+A$.

Proof: The proof of this theorem follows by going through steps which are analogous to those used by Bickel and Rosenblatt for their Propositions 2.1 and 2.2. and using the key approximation result in Theorer. 4.3. 
Theorem 4.5: Theorem 4.4 is true with $\xi_{n}(x)$ replaced by $\bar{\xi}_{n}(s)$.

Proof: Immediate from Theorem 4.4 and Lenma 4.2 .

In order to obtain the asymptotic distribution of

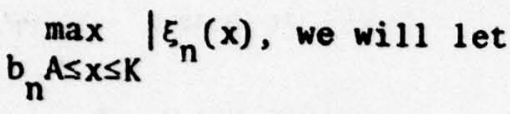

$\max _{n} A \leq x \leq K=\xi_{n}(x) \mid$ and

(4.11) $\quad \lambda(w)=\int w^{2}(t) d t$,

and

(4.12) $\quad K_{1}(w)=\frac{w^{2}(A)+w^{2}(-A)}{2 \lambda(w)}$.

If $K_{1}(w)=0$, we shall require that

(w6) $\quad \int\left(w^{-}(t)\right)^{2} d t<\infty$

(notice that (W6) implies (w5)), and set

(4.13) $\quad k_{2}(w)=\int\left(w^{-}(t)\right)^{2} d t / 2 \lambda(w)$.

Let

(4.14) $\quad c_{n}=\left(2 \log \left(K / b_{n}\right)\right)^{\frac{1}{2}}$.

Define

(4.15) $\left.\quad B_{n}=c_{n} /(\lambda / w)\right)^{\frac{1}{2}}$,

and 
(4.16) $\quad a_{n}=(\lambda(w))^{2 / 2}\left[c_{n}^{2}+\log \left(c_{n} K_{1}(w) / \sqrt{2 \pi}\right)\right] / c_{n}$

when $w$ satisfies (w1)-(W5) and $k_{1}(w)>0$,

(4.17) $\quad a_{n}=(\lambda(w))^{\frac{1}{2}}\left[c_{n}^{2}+\log \left(k_{2}(w) / \pi\right)\right] / c_{n}$

when $w$ satisfies (w1) $-\left(w^{4}\right),(w 6)$ and $k_{1}(w)=0$.

Theorem 4.6. Let $M_{n}=\max _{b_{n} A \leq x \leq K}\left|\xi_{n}(x)\right|$. Let (w1)-(w5) hold with $K_{1}(w)>0$ or (W1)-iv(4) and (W6) hold with $K_{1}(w)=0$. Let (B1), (B2), (F1) and (F2) hold. Then

(4.19) $P\left\{\beta_{n}\left(M_{n}-\alpha_{n}\right) \leq z\right\} \rightarrow e^{-2 e^{-2}}$

for $0<x<\infty$.

Proof: Follows from Theorem 4.4 and the results on the extrema of a stationary Gaussian process with an autocorrelation function $\rho(\theta)$ given in Appendix A of Bickel and Rosenblatt. Theorem 4.7. Let $i_{n}=\max _{b_{n} A \leq x \leq K}\left|\bar{\xi}_{n}(x)\right|$. Theorem 4.6 holds with $M_{n}$ replaced

Proof: Immediate from Theorems 4.5 and 4.6.

In order to obtain results which are useful for applications, we will have to replace $r_{n}^{*}(x)$ in $\xi_{n}(x)$ and $\bar{\xi}_{n}(x)$ by $r(x)$. We shall now indicate the steps which enable us to do this. We impose a further condition 
on $F$ which implies (F2) if $F(K)>0$ :

(F3)

$$
r(x) \text { is twice continuously differentiable. }
$$

Lemma 4.8. Let (F3) hold. Let (W1), (W2) hold. Then

$$
\text { (4.19) } \sup _{0 \leq x \leq K}\left|r_{n}^{*}(x)-r(x)\right| \leq L b_{n}^{2}
$$

where $L$ is some finite number.

Proof: Now,

$$
\begin{aligned}
r_{n}^{*}(x)-r(x) & =\frac{1}{b_{n}} \int w\left(\frac{x-s}{b_{n}}\right) d H(s)-r(x) \\
& =\int\left[r\left(x+t b_{n}\right)-r(x)\right] w(t) d t \\
& =\int_{-A}^{A}\left[t b_{n} r^{\prime}(x)+\frac{\left(t b_{n}\right)^{2}}{2}\left(r^{-1}(x)+r\left(x, t, b_{n}\right)\right)\right] w(t) d t
\end{aligned}
$$

where $r\left(x, t, b_{n}\right) \rightarrow 0$ uniformly in $x, t$

$$
=b_{n}^{2} r^{\rho-}(x) \int_{-A}^{A} t^{2} w(t) d t[1+0(1)],
$$

and $O(1)$ is uniform in $x$.

Thus $\sup _{0 \leq x \leq K}\left|r_{n}^{*}(x)-r(x)\right| \leq L b_{n}^{2}$ where $0 \leq L<\infty$.

Let the constants $b_{n}$ satisfy the condition

(B3)

$$
n b_{n}^{5} \log b_{n} \rightarrow 0
$$


For instance $b_{n}=n^{-a}$ with $\frac{1}{5}<a<\frac{1}{2}$ will satisfy (B1) - (B3).

Theorem 4.9. Under the additional assumption (B3) holds, we may replace $r_{n}^{*}(x)$ by $r(x)$ in the definitions of $\xi_{n}(x), \bar{\xi}_{n}(x), N_{n}$ and $\bar{M}_{n}$, and Theorems 4.6 and 4.7 will continue to hold for the new $M_{n}$ and $\bar{M}_{n}$.

Proof: Note that

$$
\begin{aligned}
& \sqrt{2 \log K / b_{n}} \sqrt{n b_{n}}\left(h_{n}^{(3)}(x)-r(x)\right) \\
& =\sqrt{2 \log K / b_{n}} \sqrt{n b_{n}}\left(h_{n}^{(3)}(x)-r_{n}^{*}(x)\right) \\
& +\sqrt{2 \log K / b_{n}} \sqrt{n b_{n}}\left(r_{n}^{*}(x)-r(x)\right) \\
& =c_{n} \sqrt{B^{-}(x)} \xi_{n}(x)+o\left(\sqrt{n b_{n}^{5} \operatorname{log~K/b}{ }_{n}}\right) \\
& =c_{n} \sqrt{B^{-}(x)} \xi_{n}(x)+o(1) .
\end{aligned}
$$

This fact proves the theorem.

Notice that the denominator $\sqrt{B^{-}(x)}(=\sqrt{r(x) / F(x)}$ in the definitions of $\xi_{n}(x)$ and $\bar{\xi}_{n}(x)$ is unknown. In Theorem 4.10 we replace $B^{-}(x)$ by an estimate to obtain uniform conficence bands for $r(x)$ using our smoothed estimator $\bar{r}_{n}(x)$. In an analogous manner, we can also obtain unifrom confidence bands for $r(x)$ using $h_{n}^{(3)}(x)$.

Theorem 4.10. Let (W1) - (W5) hold with $K_{1}(w)>0$ or let (W1) - (W4), (W6) hold with $K_{1}(w)=0$. Let (B1) - (B3), (F1), (F3) hold. Let $a_{n}$ 
and $B_{n}$ be as defined in $(4.15),(4.16)$ and $(4.17)$. Then

(4.20) $P\left\{\bar{r}_{n}(x)-\left(\frac{\bar{r}_{n}(x)}{n_{n} \bar{F}_{n}(x)}\right)^{\frac{1}{2}}\left(\frac{2}{\beta_{n}}+\alpha_{n}\right) \leq r(x)\right.$

$$
\left.s \bar{r}_{n}(x)+\left[\frac{\bar{r}_{n}(x)}{n_{n} \bar{F}_{n}(x)}\right)^{\frac{1}{2}}\left(\frac{z}{n}+a_{n}\right)\right\} \rightarrow e^{-2^{-z}},
$$

for $0<z<\infty$.

Proof: This theorem follows from the fact that $\bar{r}_{n}(x) / \bar{F}_{n}(x)$ and $h_{n}^{(3)}(x) / \bar{F}_{n}(x)$ are uniformly estimates of $B^{-}(x)$.

Remark: To obtain a 100ar uniform confidence band for $r(x)$ for $b_{n} A \leq x \leq k$ we use the two functions

$$
\bar{r}_{n}(x) \pm\left(\frac{\bar{r}_{n}(x)}{n b_{n} \bar{F}_{n}(x)}\right)^{\frac{1}{2}}\left(\frac{2}{\beta_{n}}+\alpha_{n}\right)
$$

with $z=-\log (-(1 / 2) \log \alpha)$.

\section{An Illustrative Example With Simulations.}

A smoothing window which is quite natura) ich also turns out to be computationally simple, is the uniform

(5.1) $\quad w(u)=\frac{1}{2}, \quad|u|<1$.

This w satisfies (w1) - (w5) with $K_{1}(w)=\frac{1}{2}>0$. 
In order to obtain an expression for $\bar{r}_{n}(x)$ using this window, we shall make use of the following elementary results.

For any $x \in[r-1, r]$

$$
\frac{1}{r} \leq \int_{r-1}^{r} \frac{1}{x} d x=\log \frac{r}{r-1} \leq \frac{1}{r-1}
$$

It follows from the above that

$$
\sum_{r=n-j+1}^{n-k} \frac{1}{r} \leq \log \frac{n-k}{n-j}
$$

and

$$
\sum_{r=n-j+2}^{n-k+1} \frac{1}{r-1} \geq \log \frac{n-k+1}{n-j+1} .
$$

For any $x \geq 0$, and any specified $b_{n}$, let $z_{1}=(j-1)$ be the number of observations (failures) to the left of $x-b_{n}, z_{2}=(k-j+1)$ the number of observations between $x+b_{n}$ and $x-b_{n}$, and $z_{3}=(n-k)$ be the number of observations to the right of $x+b_{n}$. Also let

$$
\frac{1}{U_{i}}=\frac{1}{(n-i+1)\left(x_{(i)}-X_{(i-1)}\right)^{\prime}} \text { for } x_{(i-1)} \leq x<x_{(i)} \text {, }
$$

$i=1,2, \ldots, n$. If $w(x)=\int_{0}^{x} w(s) d s$, then 


$$
\bar{r}_{n}(x)=\sum_{i=1}^{n} \int_{(i-1)}^{x_{(i)}} r_{n}(x) w(x-s) d s=\sum_{i=1}^{n} w\left(x-x_{(i)}\right)\left(\frac{1}{U_{i+1}}-\frac{1}{U_{i}}\right)
$$

For $w(\cdot)$ given by equation (5.1), we have

$$
\begin{aligned}
\bar{r}_{n}(x) & =\frac{1}{2 b_{n}}\left[\frac{x_{(j)}-\left(x-b_{n}\right)}{U_{j}}+\frac{x_{(j+i)}-x_{(j)}}{U_{j+1}}+\ldots+\frac{x_{(i)}-x_{(i-1)}}{U_{i}}\right. \\
& \left.+\ldots+\frac{x_{(k)}-x_{(k-1)}}{U_{k}}+\frac{x_{n}-b_{n}-x_{(k)}}{U_{k+1}}\right]
\end{aligned}
$$

or that

$$
\vec{r}_{n}(x)=\frac{1}{2 b_{n}}\left[\frac{x_{(j)}-\left(x-b_{n}\right)}{u_{j}}+\ldots+\frac{x_{(i)}-x_{(i-i)}}{(n-i+1) v_{(i)}}+\ldots+\frac{x_{n+b_{n}-x_{(k)}}}{u_{k+1}}\right]
$$

Based upon the above and the elementary results presented before, we can write

$$
\frac{1}{2 b} \sum_{n-k+1}^{n-j} \frac{1}{r} \leq \bar{r}_{n}(x) \leq \frac{1}{2 b} \sum_{n-k}^{n-j+1} \frac{1}{r} .
$$

The left hand inequality is obtained by ignoring the first and the last teras of (5.3), whereas the right hand inequality is obtained by setting $x+b_{n}=x_{(k+1)}$ and $x-b_{n}=x_{(j-1)}$ in the last and the first terms of (5.3), respectively. 
Thus

$$
\begin{aligned}
& \frac{1}{2 b_{n}} \log \frac{n-j+1}{n-k+1} \leq \bar{r}_{n}(x) \leq \frac{1}{2 b_{n}} \log \frac{n-j+1}{n-k-1} \text {, or } \\
& \frac{1}{2 b_{n}} \log \frac{z_{2}+z_{3}}{z_{3}+1} \leq \bar{r}_{n}(x) \leq \frac{1}{2 b_{n}} \log \frac{z_{2}+z_{3}}{z_{3}-1} .
\end{aligned}
$$

In view of the above, a computationally simple expression for $\bar{r}_{n}(x)$ is

$$
\bar{x}_{n}(x) \approx \frac{1}{2 b_{n}} \log \frac{z_{2}+z_{3}}{z_{3}}
$$

\subsection{Results of a Monte-Carlo Experiment.}

In this section we shall summarize our experience with the estimator given by equation (5.4), based on a Monté Carlo experiment.

Random samples of size $n(=10,15,20$ and 50$)$ were generated from an exponential distribution with scale parameter 1.0 , and a Weibull distribution with shape parameter 2.0, respectively. For each sample size we obtain $\bar{r}_{n}(x)$ using a uniform window with $b_{n}=\frac{1}{n^{a}}$, for $a=0.05,0.10,0.15$, $0.20,0.25$, and 0.75 . We repeat this procedure 500 times for each of the specified 24 combinations of sample size and window width. Based upon the results of these 500 trials, we calculate at each point $x$, the average, the standard error, and the root mean square error of $\bar{r}_{n}(x)$. These statistics 
are summarized in Table 5.1 for the exponential distribution at the point $x=1.0$, and in Table 5.2 for the Weibull distribution at the point $x=0.5$. In Figure 5.1 we show a plot of the average value of $\bar{r}_{n}(x)$ at each point $x$, based on $n=10$ and $a=0.75$ for the exponential distribution, whereas in Figure 5.2 we show a similar plot for $n=50$. In Figures 5.3 and 5.4 we give plots analogous to those in Figures 5.1 and 5.2, respectively, but for the Weibull distribution.

On the basis of the above described experiment, we can state the following by way of conclusion:

i) For samples as small as 10 or 15, the smoothed estimator obtained by using windows of small width is generally unbiased; the bias increases with the width of the window. This phenomenon is especially true for points in the aiddle of the range of $x$.

ii) The standard error decreases with the width of the window.

iii) It appears that towards the end points of the range of $x, a$ smoothed estimator based on a narrower window width performs better than one based on a wider window width.

The above comments suggest the possibility of using windows of varying widths. A version of this strategy has been considered by Miller and Singpurwalla (1978). For further details on the Monte-Carlo experiment, we refer the reader to Chandra (1977). 
TABLE 5.1

SAMPLING PROPERTIES OF SMDOTHED ESTIMATOR OF THE FAILURE RATE AT THE POINT $x=1.0$ FRON AN EXPONENTIAL DISTRIBUTION

\begin{tabular}{|c|c|c|c|c|}
\hline $\begin{array}{l}\text { SAMPLE } \\
\text { SIZE }\end{array}$ & WINDOW WIDTH & MEAN & STANDARD ERROR & $\begin{array}{c}\text { ROOT MEAN } \\
\text { SQUARED ERROR }\end{array}$ \\
\hline \multirow[t]{6}{*}{10} & 0.356 & 1.0649 & 1.0259 & 1.0280 \\
\hline & 1.125 & 0.9362 & 0.5126 & 0.5166 \\
\hline & 1.262 & 0.9178 & 0.4854 & 0.4923 \\
\hline & 1.416 & 0.8518 & 0.4517 & 0.4754 \\
\hline & 1.589 & 0.8240 & 0.4608 & 0.4932 \\
\hline & 1.782 & 0.7710 & 0.4396 & 0.4961 \\
\hline \multirow[t]{6}{*}{15} & 0.262 & 1.1780 & 1.1004 & 1.1147 \\
\hline & 1.016 & 1.0373 & 0.5153 & 0.5167 \\
\hline & 1.164 & 1.0248 & 0.4376 & 0.4383 \\
\hline & 1.332 & 1.0452 & 0.4327 & 0.4350 \\
\hline & 1.526 & 0.9704 & 0.3974 & 0.3985 \\
\hline & 1.747 & 0.9306 & 0.4019 & 0.4079 \\
\hline \multirow[t]{6}{*}{20} & 0.212 & 1.0978 & 0.8748 & 0.8802 \\
\hline & 0.946 & 1.0537 & 0.4501 & 0.4533 \\
\hline & 1.099 & 1.0751 & 0.4123 & 0.4191 \\
\hline & 1.276 & 1.0353 & 0.3658 & 0.3675 \\
\hline & 1.582 & 1.0376 & 0.3606 & 0.3626 \\
\hline & 1.722 & 0.9966 & 0.3452 & 0.3452 \\
\hline \multirow[t]{6}{*}{50} & 0.106 & 1.0145 & 0.7258 & 0.7259 \\
\hline & 0.752 & 1.0237 & 0.2835 & 0.2845 \\
\hline & 0.915 & 1.0183 & 0.2599 & 0.2605 \\
\hline & 1.112 & 1.0479 & 0.2449 & 0.2495 \\
\hline & 1.353 & 1.0280 & 0.2284 & 0.2301 \\
\hline & 1.645 & 1.0251 & 0.1982 & 0.1998 \\
\hline
\end{tabular}


TABLE 5.2

SAMPLING PROPERTIES OF SMOOTHED ESTIMATOR OF THE FAILURE RATE AT THE POINT $x=0.5$ FROM A WEIBULL DISTRIBUTION

\begin{tabular}{|c|c|c|c|c|}
\hline $\begin{array}{l}\text { SAMPLE } \\
\text { SIZE }\end{array}$ & WINDOW WIDTH & WEAN & STANDARD ERROR & $\begin{array}{l}\text { ROOT NEAN } \\
\text { SQUARED ERROR }\end{array}$ \\
\hline \multirow[t]{6}{*}{10} & 0.356 & 1.0459 & 0.6402 & 0.6419 \\
\hline & 1.125 & 1.0769 & 0.4614 & 0.4678 \\
\hline & 1.262 & 1.0314 & 0.4598 & 0.4609 \\
\hline & 1.416 & 0.9773 & 0.4189 & 0.4195 \\
\hline & 1.589 & 0.9309 & 0.4644 & 0.4695 \\
\hline & 1.782 & 0.8130 & 0.4744 & 0.5099 \\
\hline \multirow[t]{6}{*}{15} & 0.262 & 1.0530 & 0.5870 & 0.5894 \\
\hline & 1.016 & 1.0746 & 0.4022 & 0.4091 \\
\hline & 1.164 & 1.0649 & 0.3874 & 0.3928 \\
\hline & 1.332 & 1.0995 & 0.3783 & 0.3912 \\
\hline & 1.526 & 1.0549 & 0.3818 & 0.3857 \\
\hline & 1.747 & 1.0063 & 0.4144 & 0.4144 \\
\hline \multirow[t]{6}{*}{20} & 0.212 & 0.9351 & 0.5809 & 0.5811 \\
\hline & 0.946 & 1.0422 & 0.3224 & 0.3251 \\
\hline & 1.099 & 1.0633 & 0.3245 & 0.3307 \\
\hline & 1.276 & 1.0494 & 0.2945 & 0.2987 \\
\hline & 1.582 & 1.0855 & 0.3350 & 0.3458 \\
\hline & 1.722 & 1.0816 & 0.3479 & 0.3574 \\
\hline \multirow[t]{6}{*}{50} & 0.106 & 0.9893 & 0.4915 & 0.4917 \\
\hline & 0.752 & 1.0270 & 0.2043 & 0.2061 \\
\hline & 0.915 & 1.0230 & 0.1997 & 0.2010 \\
\hline & 1.112 & 1.0154 & 0.1912 & 0.1918 \\
\hline & 1.353 & 1.0339 & 0.1958 & 0.1988 \\
\hline & 1.645 & 0.0867 & 0.1876 & 0.2067 \\
\hline
\end{tabular}




\section{REFERENCES}

Ahmad, I. and Lin, Pi-Erh (1977). Nonparametric estimation of a vector-valued bivariate failure rate. The Annals of Statistics 5 1027-1038.

Bickel, P. J. and Rosenblatt, N. (1973). On some global measures of the deviations of density function estimates. The Annals of Statistics, Vo1. 1, No. 6, 1071-1095.

Chandra, M. (1977). Small Sample Behavior of a smoothed estimator of the failure rate function - A Mont6-Carlo Investigation. Technical Memorandum, Serial TM-66506.

Feller, W. (1966). An Introduction to Probability Theory and its Applications. Fol. II. John Wiley and Sons, Inc., New York.

Komlos, J., Hajor, P. and Tusnady, G. (1975). An approximation of partial suns of independent $\mathrm{rv}$ 's and the sample df. I.Z. Wahrscheinlichkeitstheorie und Verw. Gebiete 32., 111-131.

Miller, D. R. and Singpurwalla, N. D. (1977). Failure rate estimation using random smoothing. Technical Paper, Serial T-347, Program in Logistics, The George Washington University.

Rice, J. and Rosenblatt, M. (1976). Estimation of the $\log$ survivor function and hazard function. Sankhya, Series A, Vol. 38, Part 1, 60-78.

Rosenblatt, M. (1976). On the maximal deviation of $k$-dimensional density estimates. The Annals of Probability, Vol. 4, No. 6, 1009-1015.

Shaked, Moshe (1978). An estimator for the generalized end rate function, University New Mexico Technical Report No. 343.

Singpurwalla, N. D. (1975). Time series analysis of failure rate processes. Reliability and Fault Tree Analysis, SIAM, Philadelphia. 483-507. 


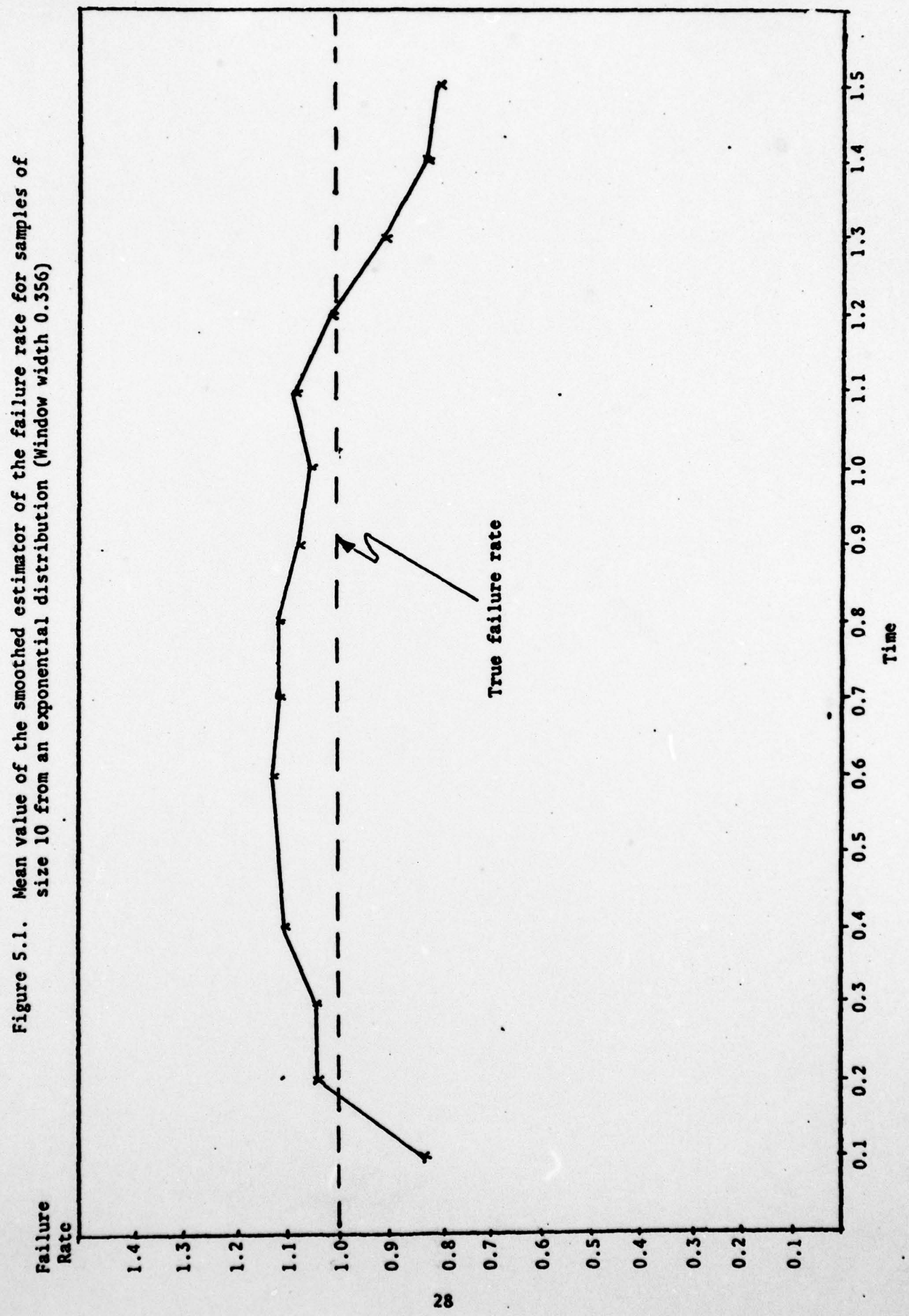




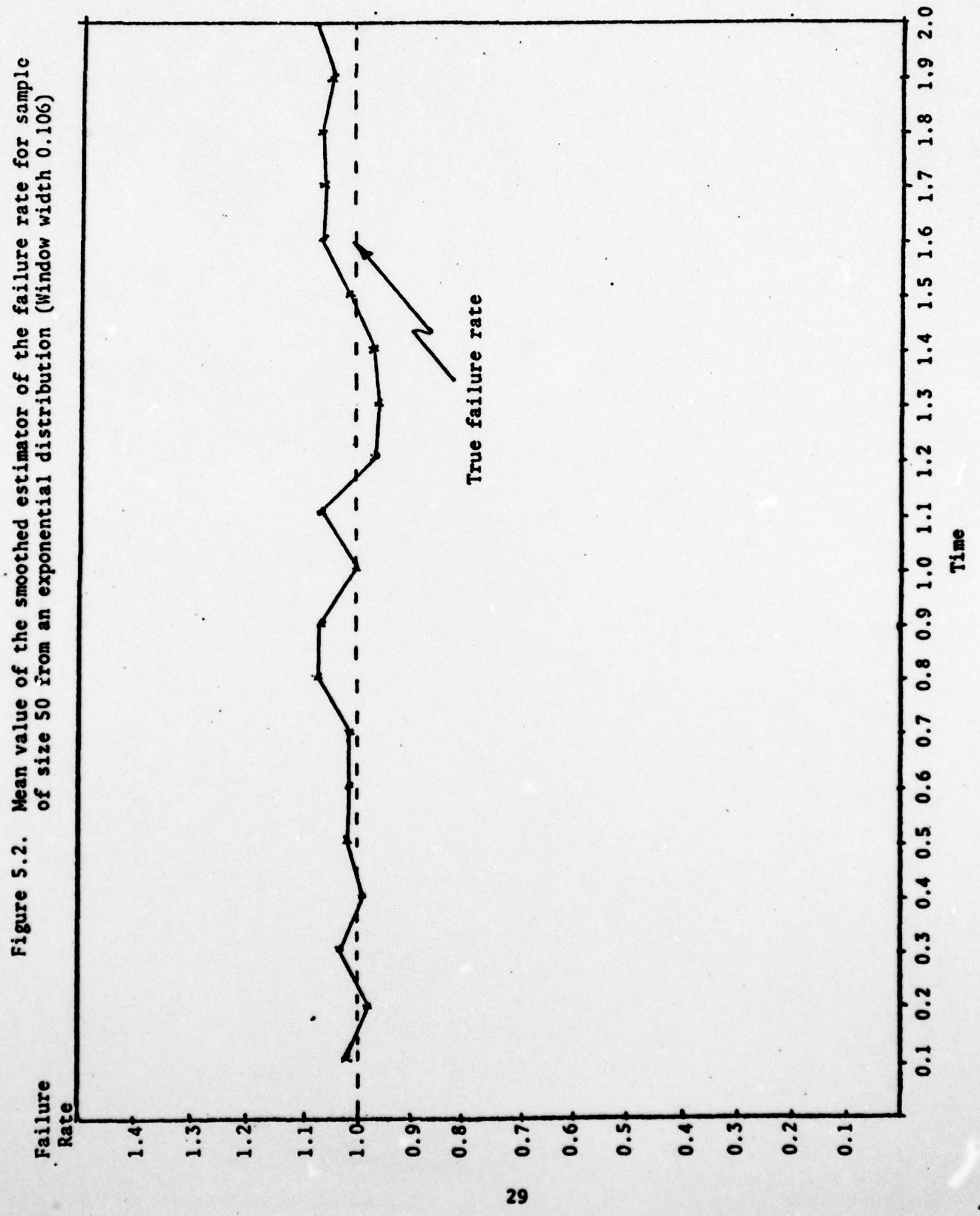




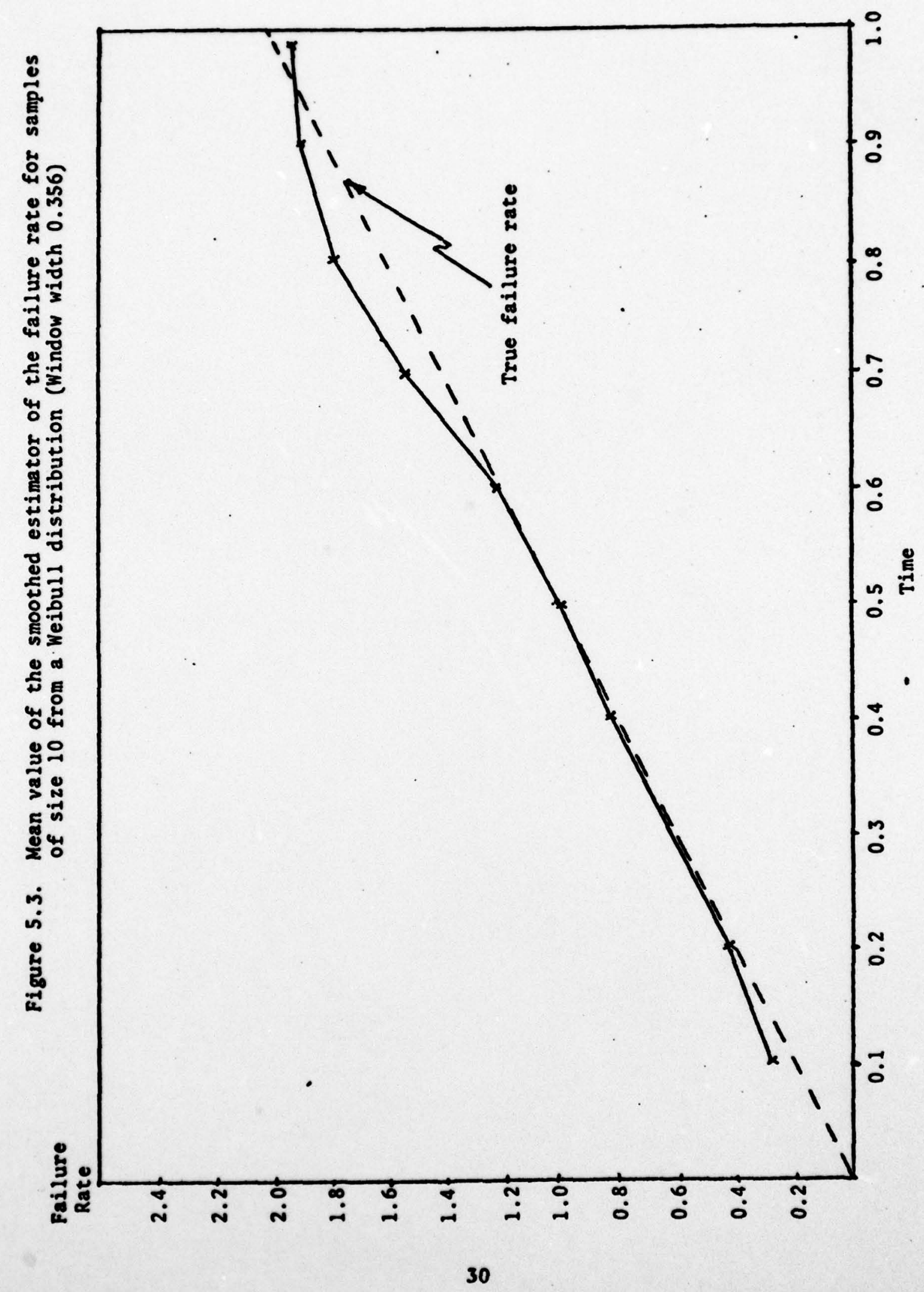




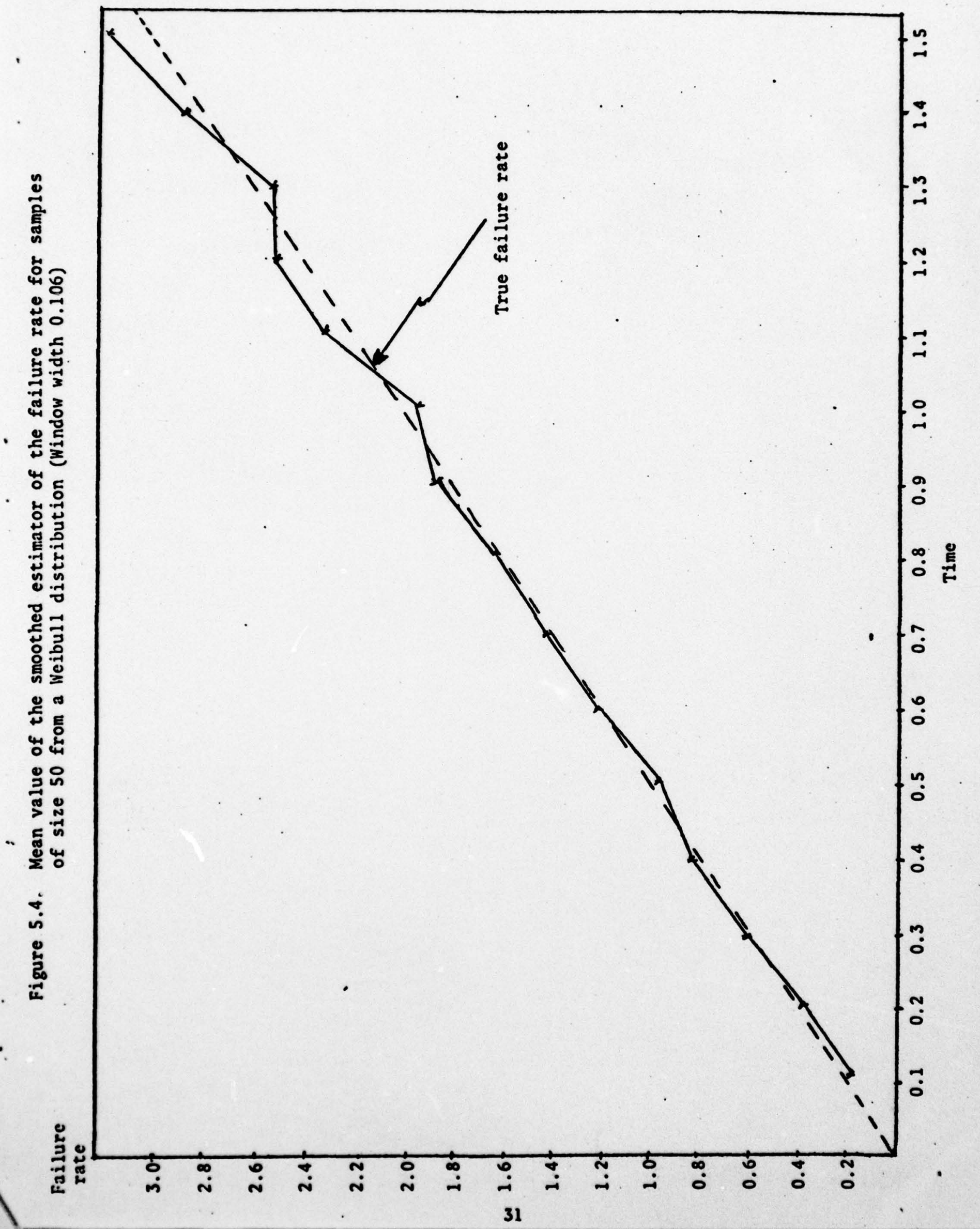

